Psychological Medicine

http://journals.cambridge.org/PSM

Additional services for Psychological Medicine:

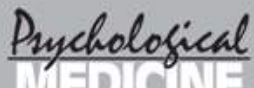

Email alerts: $\underline{\text { Click here }}$

Subscriptions: Click here

Commercial reprints: Click here

Terms of use : $\underline{\text { Click here }}$

\title{
Online randomized controlled trial of brief and full cognitive behaviour therapy for depression
}

\author{
H. CHRISTENSEN, K. M. GRIFFITHS, A. J. MACKINNON and KYLIE BRITTLIFFE
}

Psychological Medicine / Volume 36 / Issue 12 / December 2006, pp 1737 - 1746

DOI: 10.1017/S0033291706008695, Published online: 29 August 2006

Link to this article: http://journals.cambridge.org/abstract_S0033291706008695

How to cite this article:

H. CHRISTENSEN, K. M. GRIFFITHS, A. J. MACKINNON and KYLIE BRITTLIFFE (2006). Online randomized controlled trial of brief and full cognitive behaviour therapy for depression.

Psychological Medicine, 36, pp 1737-1746 doi:10.1017/S0033291706008695

Request Permissions : $\underline{\text { Click here }}$ 


\title{
Online randomized controlled trial of brief and full cognitive behaviour therapy for depression
}

\author{
H. CHRISTENSEN*, K. M. GRIFFITHS, A. J. MACKINNON \\ AND K YLIE BRITTLIFFE \\ Centre for Mental Health Research, The Australian National University, Canberra, Australia
}

\begin{abstract}
Background. Effective internet-based programs for depression usually incorporate a component that provides telephone or email contact. Open access websites, without such contact, show high rates of attrition and poorer outcomes. The present study was designed as an exploratory investigation of the parameters that influence the effectiveness and retention of users on open access websites. We investigated whether brief cognitive behaviour therapy (CBT) was as effective as an extended version, whether add-on components of behaviour therapy or stress management contributed to positive outcomes, and whether longer programs were associated with greater attrition.
\end{abstract}

Method. An online randomized controlled trial (RCT) was conducted between 13 January 2005 and 26 May 2005 (19 weeks). A total of 2794 registrants (1846 women and 948 men; median age category 35-44 years) with elevated scores on the Goldberg Depression Scale of 5.96 (S.D. $=2 \cdot 09$ ) elected online to be randomized to one of six versions of a CBT website. The versions were compiled consisting of various components of brief CBT, extended CBT, behaviour strategies, stress management and problem solving.

Results. A total of $20 \cdot 4 \%$ of participants completed the assigned intervention. The interaction of measurement occasion and treatment version was significant $[F(13,131)=2 \cdot 20, p=0 \cdot 01]$. A single module of brief introductory CBT was not effective in reducing depression symptoms. However, extended CBT with or without the addition of behaviour strategies resulted in the reduction of depression.

Conclusions. Brief CBT-based interventions are not as effective as extended interventions. However, longer programs are associated with higher rates of dropout.

\section{INTRODUCTION}

Cognitive behaviour therapy (CBT) programs for depression have been successfully delivered on the internet (Christensen et al. 2004a; Griffiths et al. 2004; Clarke et al. 2005), supporting the feasibility of internet-based public health approaches to depression. However, successful programs usually incorporate some telephone or email contact. Adherence to the web application appears to be improved by

\footnotetext{
* Address for correspondence: Professor Helen Christensen, Centre for Mental Health Research, The Australian National University, Canberra ACT 0200, Australia.

(Email: Helen.Christensen@anu.edu.au)
}

contact, support or by tracking by an interviewer or counsellor. For example, Clarke et al. (2005) compared the outcomes of two trials of the ODIN website and found that reminders were the crucial factor in determining effectiveness and retention. Similarly, research using a CBT website (MoodGYM) reported higher retention rates in those provided with weekly tracking compared to those accessing the site directly from the world wide web (Christensen et al. 2004b). Attrition from open access nontracked websites can be very high, with as few as $1 \%$ of users completing a full course of online therapy (Eysenbach, 2005). Nevertheless, these websites have great potential in addressing a 


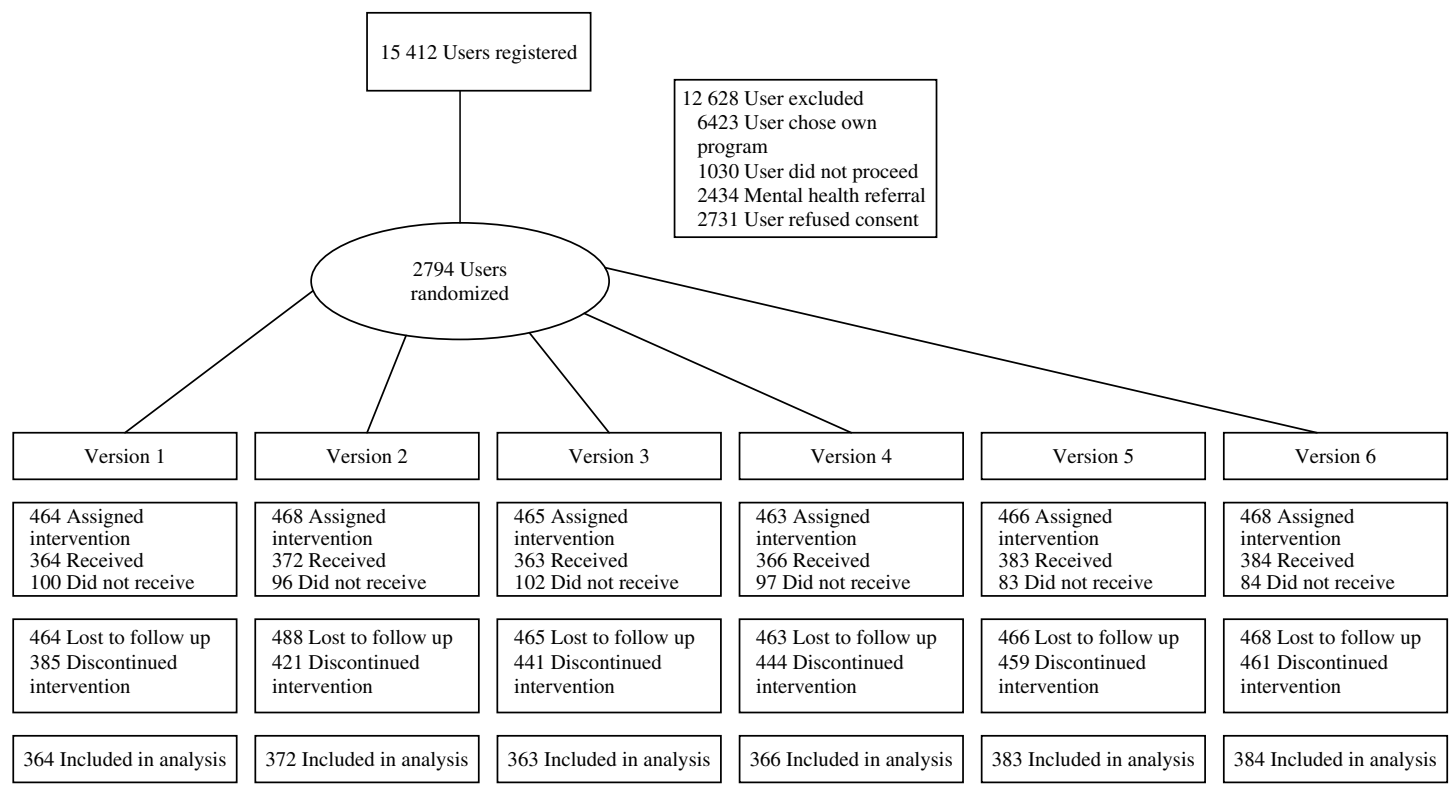

Fig. 1. Flow diagram.

large proportion of unmet need. More research is required to investigate methods to optimize outcomes for these potentially low-cost interventions.

The present study was designed as an investigation of the parameters that influence open access website use and retention. Using a randomized controlled trial (RCT), we investigated whether a brief CBT was as effective as an extended CBT version, whether additional components of behaviour therapy, problem solving or stress management improved depression outcomes, and whether longer programs were associated with greater attrition. This was achieved by constructing six versions of an online CBT intervention using material from the MoodGYM site. These six versions incorporated components of brief CBT, extended CBT, stress management, problem solving and behaviour therapy. These configurations were designed to permit the evaluation of the importance of the content of each module while preserving site functionality. Study participants were spontaneous visitors to the open website and were recruited directly to the trial after first providing consent. Randomization to one of the six conditions was based on an algorithm. Previous studies of MoodGYM have focused on efficacy outcomes from an RCT that recruited participants from the community, provided telephone contact and used mail surveys to collect data (Christensen et al. 2004a; Griffiths et al. 2004), or on naturalistic observations of online visitors (Christensen et al. 2002, 2004 b). This study is a new effectiveness trial of six versions the MoodGYM website. The website is an open access, free website on the world wide web.

\section{METHOD}

\section{Participants}

Participants were recruited between 13 January 2005 and 26 May 2005, randomized using automated procedures (see Fig. 1); 61.7\% logged in from Australia, 12.6\% from the UK, $10.0 \%$ from the USA, $4.5 \%$ from Canada and $2 \cdot 5 \%$ from New Zealand.

\section{Recruitment and allocation}

Spontaneous visitors to the site were asked whether they would participate in a research project designed to investigate the usefulness of different versions of the site. There was no concealment of the randomization conditions. A full description of the six versions was provided. Following the procedures described by Ward et al. (2000), participants were then able 
to choose whether they preferred to receive one of the six conditions or whether they were prepared to be randomized to one of these. Participants who reported that their clinician had referred them to the site were excluded from randomization. Apart from preference not to be randomized or current self-reported referral from a clinician, there were no exclusion criteria.

\section{Design}

The protocol was approved by the Human Ethics Committee of the Australian National University. For those who chose to be randomized, an algorithm was used that ensured an equal allocation of users across conditions in each of four groups stratified by sex and age (under 30 years, 30 years and over). Each group was uniformly allocated to each treatment condition by assigning each of the conditions cyclically but in random order within the cycle. Consent was obtained by the participant pressing the 'I agree' button on the bottom of the web page describing the aims of the trial and outlining the range of potential interventions. Participants were assessed at baseline (directly before commencing Module 1), at the commencement of each subsequent module and immediately after their last assigned module (endpoint). Participants created pseudonyms at registration to allow identification on subsequent visits. The participant's version of the program was made available immediately. This allowed the users to complete the program at their own pace. Once a module was completed, the participant's responses were saved and protected from future modification. While pages of the modules could be revisited and previous scores displayed, participants were not able to repeat assessments (see outcome measures below). There was a final assessment at the end of all six versions. There were no trial managers and no direct or indirect communication from the researchers. All trial procedures were automated. All assessments were undertaken online.

\section{Interventions}

The complete MoodGYM program consists of five 20- to 40-minute modules undertaken sequentially. Module 1 introduces six fictional site characters, gives feedback on anxiety and depression scores, provides an introduction to the core concepts of CBT, the program worksheets and the interactive online workbook. Module 2 describes 'warpy thoughts' (Parslow et al. 2006), methods to contest them, central areas of vulnerability and provides online exercises that train users to identify dysfunctional thoughts and modify them. Module 3 provides a set of behavioural strategies to counter the negative feelings of anxiety and depression. These include exercises to improve self-esteem, to decrease egocentric interpretations and to increase social and physical activity using tools such as the Pleasant Events Schedule. Module 4 provides information about stress, assessments to measure and provide feedback about life events, tools to cope with family and relationships, and downloadable relaxation tapes. Module 5 provides simple problem-solving skills in the context of coping with family and peer relationships. It also provides some basic information about co-morbidity.

For this trial, the full MoodGYM program was configured into six separate online versions of the program based on the five modules. Version 1 (Brief CBT) included Module 1 only, Version 2 comprised Modules 1 and 5 (Brief CBT; Problem Solving), Version 3 included Modules 1, 4 and 5 (Brief CBT, Stress and Problem Solving), Version 4 provided Modules 1, 2 and 5 (Extended CBT and Problem Solving), Version 5 incorporated Modules 1, 2, 3 and 5 (Extended CBT, Behavioural Strategies and Problem Solving) and Version 6 (Full Program) included all modules. These configurations were designed to permit the evaluation of the importance of the content of each module while preserving site functionality and offering a defensible, cohesive evidence-based intervention to all participants.

\section{Outcome measures}

The primary outcome measure was score on the Goldberg Depression Scale (Goldberg et al. 1988; Christensen et al. 1999). The items were derived by latent trait analysis from a standardized psychiatric research interview (Goldberg et al. 1988) and correlate strongly with items from the observer-based Hamilton Depression Scale (Holm et al. 2001). Retention in the program and score on the Goldberg Anxiety Scale were secondary outcomes. The Goldberg scales consist of nine depression and nine anxiety 
items. The items are coded 0 (no) or 1 (yes), with participants reporting these responses to 'some specific questions about your health and how you are feeling in the past month'. These scales were chosen because the item content is closely aligned to the symptomatology underpinning DSM and ICD classifications but the scales are brief enough to repeatedly administer in the course of a therapeutic intervention. In addition, norms for the scales in an epidemiological survey (Parslow et al. 2004) were available. The Depression and Anxiety Scales were completed at the beginning of the trial, at the start of each subsequent module and at the end of each program.

\section{Statistical analysis}

Demographic and retention differences between conditions were investigated using $\chi^{2}$ tests. Analyses of outcome data were undertaken on a modified intent-to-treat (ITT) basis: all participants were included in the analyses except for those who failed to complete pretest assessments after randomization and for whom no data were available. Design factors were measurement occasion (baseline and prior to subsequent and intervention endpoint) and treatment version (the modules the participant was assigned). In this design, absence of assessments before modules not administered due to the incomplete factorial nature of the design represents planned missingness. Because of the design of the current MoodGYM site, there were no intermittent missing data; participants could not continue to their next assigned module without completing an assessment. Thus missingness due to attrition followed a monotone pattern.

Mixed model repeated-measures analysis of variance (ANOVA) was undertaken using SAS version 9.1 PROC MIXED using an unstructured covariance matrix. This yields unbiased estimates of ITT effect under the assumption that data from participants who withdrew were missing at random (MAR). MAR allows that the mechanism of missingness may be related to observed variables such as previous depression assessments, but is unrelated to the value of the missing observation(s).

In addition to the principal ITT analyses, secondary analysis restricted to those participants who completed their assigned modules was also undertaken.

\section{RESULTS}

\section{Flow chart and participants}

A total of 15412 registered on site between 13 January 2005 and 26 May 2005 (19 weeks), with 2731 refusing consent $(18 \%)$ and a further 1030 failing to proceed to consent by closing their web browsers $(8 \%)$. A total of $6423(42 \%)$ users chose their own program (that is, they chose which condition they preferred to undertake from the six options available), and this excluded them from the randomization component of the trial. In addition, 2434 (16\%) reported that they were recommended to the site by their clinician and were thus directed to the standard MoodGYM program. This left 2794 participants who were randomized to the six conditions (18\%) (see Fig. 1).

\section{Depression}

The six study groups did not differ on any key baseline characteristic (see Table 1). Most participants were women $(66 \%)$, and were aged 25 to 44 years. Nearly half $(42 \cdot 6 \%)$ lived in a capital city, with approximately $20 \%$ located in a remote or rural setting. Baseline depression and anxiety scores were elevated relative to the population norms, being over 1 standard deviation above that of community groups. Goldberg Depression Scores for an epidemiological sample suggest an average of 2.9 (S.D. $=2 \cdot 4$ ) for 20 - to 24 -year-olds and $2 \cdot 4$ (s.D. $=$ $2 \cdot 4)$ for 40- to 44-year-olds compared to $6 \cdot 0$ (s.D. $=2 \cdot 3)$ in those who entered the study (Parslow et al. 2004). Goldberg Anxiety Scores for the current sample ranged from $6 \cdot 1$ (s.D. $=$ $2 \cdot 2$; Version 1 ) to $5 \cdot 8$ (s.D. $=2 \cdot 2$; Version 2 ).

Least square means for Goldberg Depression Scores over time for each version of the program are shown in Fig. 2. The interaction of measurement occasion and treatment version was significant $[F(13,131)=2 \cdot 20, p=0 \cdot 0126]$. Depression scores were lower for those who underwent Version 4 (Modules 1, 2, and 5) and Version 5 (Modules 1, 2, 3 and 5). Both of these interventions included both extended and brief CBT. With the exception of the All Modules condition, those interventions that did not involve extended CBT resulted in less change. Relative to Module 1, effect sizes were $0 \cdot 20$ for Version 2 (Modules 1 and 5), 0.22 for Version 3 (1, 4 and 5), 0.40 for Version 4 
Table 1. Baseline and post-test characteristics

\begin{tabular}{|c|c|c|c|c|c|c|}
\hline Variable & $\begin{array}{l}\text { Version } 1 \\
(n=464)\end{array}$ & $\begin{array}{l}\text { Version } 2 \\
(n=468)\end{array}$ & $\begin{array}{l}\text { Version } 3 \\
(n=465)\end{array}$ & $\begin{array}{l}\text { Version } 4 \\
(n=463)\end{array}$ & $\begin{array}{l}\text { Version } 5 \\
(n=466)\end{array}$ & $\begin{array}{l}\text { Version } 6 \\
(n=468)\end{array}$ \\
\hline Women, $n(\%)$ & 307 (66) & $308(66)$ & $306(66)$ & 307 (66) & $308(66)$ & $310(66)$ \\
\hline $\begin{array}{l}\text { Age, } n(\%) \\
\text { Under } 25 \\
25-44 \\
\text { Over } 45\end{array}$ & $\begin{array}{l}115(25) \\
236(51) \\
113(24)\end{array}$ & $\begin{array}{l}139(30) \\
209(45) \\
120(26)\end{array}$ & $\begin{array}{l}120(26) \\
230(50) \\
115(25)\end{array}$ & $\begin{array}{l}134(29) \\
201(43) \\
128(28)\end{array}$ & $\begin{array}{l}124(27) \\
239(51) \\
103(22)\end{array}$ & $\begin{array}{l}133(28) \\
224(48) \\
111(24)\end{array}$ \\
\hline $\begin{array}{l}\text { Missing } \\
\text { Capital city, } n(\%)\end{array}$ & $193(42)$ & $187(40)$ & $220(47)$ & $181(40)$ & $212(46)$ & $196(42)$ \\
\hline $\begin{array}{l}\text { Education level } \\
\text { Primary } \\
\text { Secondary } \\
\text { Tertiary }\end{array}$ & $\begin{array}{c}13(3) \\
206(46) \\
230(51)\end{array}$ & $\begin{array}{c}22(5) \\
184(41) \\
239(54)\end{array}$ & $\begin{array}{c}10(2) \\
202(44) \\
247(54)\end{array}$ & $\begin{array}{c}5(1) \\
202(44) \\
247(54)\end{array}$ & $\begin{array}{c}11(3) \\
198(45) \\
232(9)\end{array}$ & $\begin{array}{c}13(3) \\
206(46) \\
230(51)\end{array}$ \\
\hline $\begin{array}{l}\text { Missing } \\
\text { Ever been depressed } \\
\text { Pretest } \\
\text { depression } \\
\text { Post-test } \\
\text { depression }\end{array}$ & $\begin{array}{l}380(83) \\
6 \cdot 0(2 \cdot 0) \\
\mathbf{3 6 4} \\
5 \cdot 8(2 \cdot 2) \\
\mathbf{7 9}\end{array}$ & $\begin{array}{c}1 \\
394(84) \\
5 \cdot 8(2 \cdot 2) \\
372 \\
5 \cdot 1(2 \cdot 8) \\
47\end{array}$ & $\begin{array}{l}387(83) \\
6 \cdot 0(2 \cdot 1) \\
\mathbf{3 6 2} \\
5 \cdot 00(2 \cdot 6) \\
\mathbf{2 4}\end{array}$ & $\begin{array}{l}371(80) \\
5 \cdot 8(2 \cdot 3) \\
366 \\
4 \cdot 1(3 \cdot 0) \\
19\end{array}$ & $\begin{array}{l}384(82) \\
\quad 6 \cdot 0(2 \cdot 0) \\
383 \\
\quad 4 \cdot 46(2 \cdot 1) \\
7\end{array}$ & $\begin{array}{l}383(82) \\
\quad 6 \cdot 0(2 \cdot 0) \\
384 \\
5 \cdot 8(2 \cdot 8) \\
7\end{array}$ \\
\hline
\end{tabular}

Values expressed as mean (s.D.) unless indicated otherwise.

No significant differences between conditions at baseline.

Bold values indicate number of participants who provided data to the specific observation.

Table 2. Number and percentage of individuals completing each assessment as a function of intervention type

\begin{tabular}{|c|c|c|c|c|c|c|}
\hline Program & Pretest & Module 2 & Module 3 & Module 4 & Module 5 & Post-test \\
\hline Version 1 & 364 & & & & & $90(25 \%)$ \\
\hline Version 2 & 372 & & & & $103(28 \%)$ & $103(28 \%)$ \\
\hline Version 3 & 362 & & & $99(27 \%)$ & $36(10 \%)$ & $99(27 \%)$ \\
\hline Version 4 & 372 & $103(28 \%)$ & & & $30(8 \%)$ & $103(28 \%)$ \\
\hline Version 5 & 383 & $95(25 \%)$ & $20(5 \%)$ & & $10(3 \%)$ & $95(25 \%)$ \\
\hline Version 6 & 384 & $90(23 \%)$ & $26(7 \%)$ & $10(3 \%)$ & $9(2 \%)$ & $90(23 \%)$ \\
\hline Total & 2231 & $288(13 \%)$ & $46(2 \%)$ & $109(5 \%)$ & $188(8 \%)$ & $569(26 \%)$ \\
\hline
\end{tabular}

(1,2 and 5), and 0.34 for Version 5 (Modules 1 , 2,3 and 5).

Contrasts between changes from baseline to endpoint were calculated between conditions. Only Versions 2 and 6 were not significantly superior to Version 1. Consistent with means shown in Fig. 2, changes from baseline to endpoint for Versions 4 and 5 were highly significantly different than for Version $1(p=0.0003$ and $p=0.0028)$. Version 4 was also superior to Versions 2 and 6, although at more marginal $p$ values $(0 \cdot 01$ to $0 \cdot 049)$.

We were also interested in examining whether completing an extra module of CBT (Module 2) produced a better outcome than simply completing three modules, regardless of their content. While change was greater for Version 4 (which included two modules of CBT) than
Version 3 (which included only one module of CBT), this was not statistically significant $(p=0 \cdot 136)$. The proportion of individuals meeting a clinical level diagnosis [an estimate based on scoring in the top $10 \%$ of scores (6 or above on the Goldberg Depression Scale)] ranged between 0.6 and 0.7 at pretest for the versions. At endpoint the values were $0.63,0.57$, $0 \cdot 50,0 \cdot 42,0 \cdot 14$ and 0.43 for Versions $1,2,3,4,5$ and 6 respectively.

We also undertook a completer analysis of the data, which included only those that finished the program and which controlled for pretest depression. There was a significant interaction of treatment version and measurement occasion $[F(5,176)=4 \cdot 47, p<0 \cdot 0007]$. Pairwise comparisons of the versions indicated significant differences, and a pattern of findings similar to those 


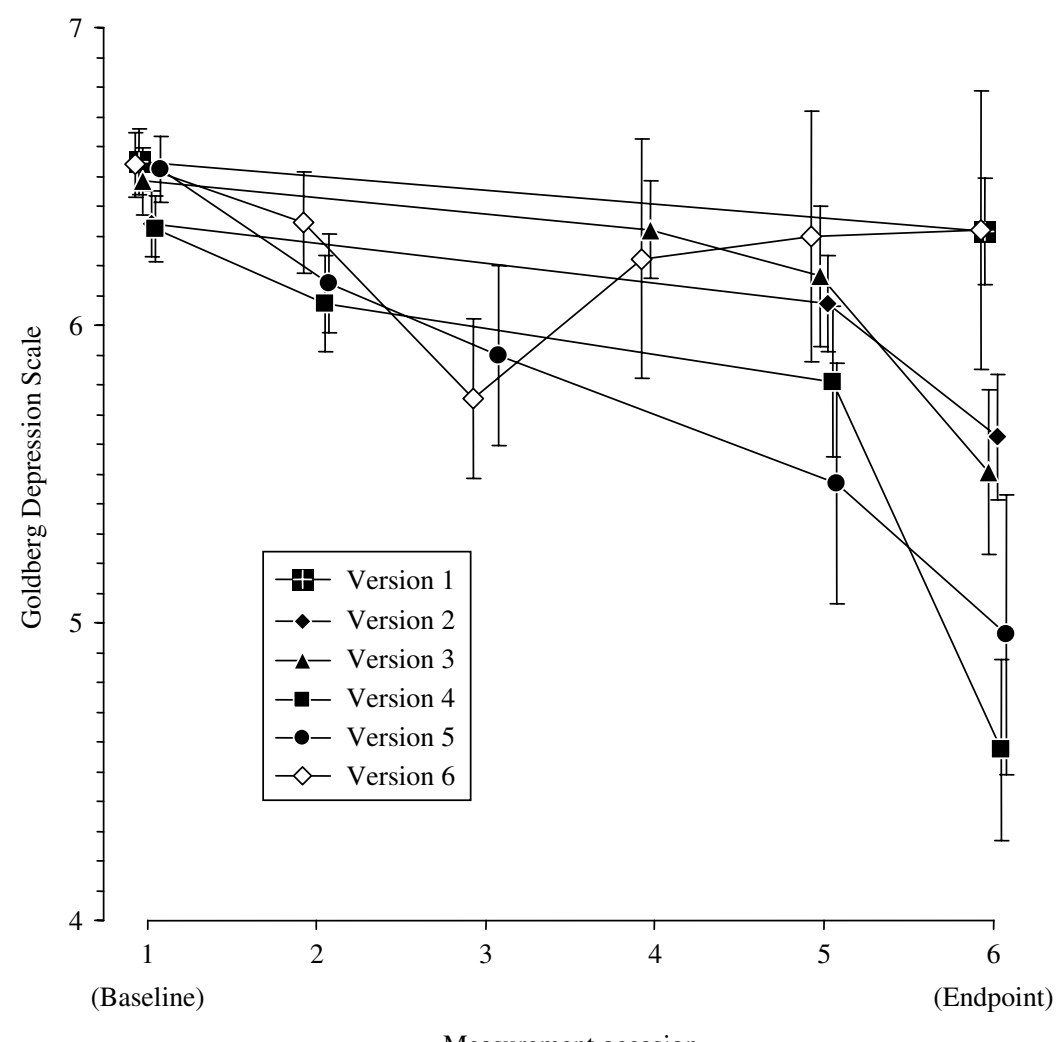

FIG. 2. Least squares means for Goldberg Depression Scale as a function of measurement occasion completed.

for the ITT analysis. The longest intervention was significantly worse than Version 4 or 5 . Version 4 (Modules 1, 2 and 5) did not differ significantly from a version with an additional module (Version 5: Modules 1, 2, 3 and 5).

\section{Anxiety}

The general pattern of change over time for the Goldberg Anxiety Scale was similar to that for the Depression Scale. However the omnibus interaction between measurement occasion and treatment version was not significant $[F(13,75 \cdot 7)=1 \cdot 21, p=0 \cdot 2899]$.

\section{Completion and retention}

There were no significant differences between conditions in the percentage of participants who agreed to be randomized but who did not complete a pretest assessment $\left(\chi^{2}=4 \cdot 94, \mathrm{df}=5\right.$, $p=0.423)$. As for ITT analysis of depression outcome, subsequent analyses of completion and retention rates were contingent on completion of a pretest assessment. In this study, completion refers to retention (see Table 2). Retention can be investigated as a function of the specific module taken or as a function of the number of modules completed, regardless of their content. The latter approach was adopted. Figure 3 show percentages of participants assigned to each program who returned to begin the next module. Between $22 \%$ and $28 \%$ of those who completed a baseline assessment returned after Module 1 and completed the next depression assessment. There was no significant association between condition and likelihood of return $\left(\chi^{2}=6 \cdot 65, \mathrm{df}=5, p=0 \cdot 248\right)$. Retention to a third session was related to version of the program $\left(\chi^{2}=15.93, \mathrm{df}=4, p=0 \cdot 003\right)$. However, this was due principally to the high rate of participants returning to complete their post-test assessment after finishing Module 5 of Version 2 and thus completing the program. The same pattern was found for completion of a fourth module $\left(\chi^{2}=11 \cdot 00, \mathrm{df}=3, p=0 \cdot 012\right)$, 


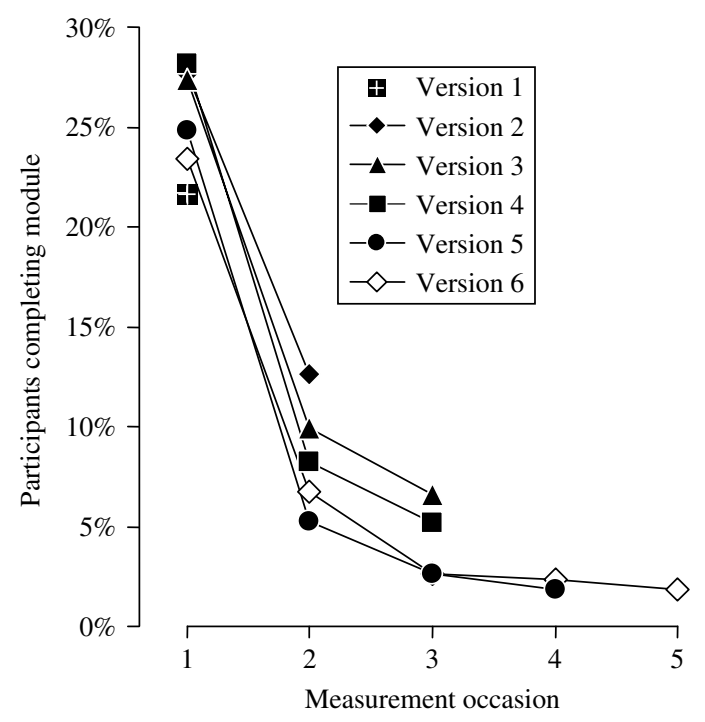

FIG. 3. Percentage of participants retained to start next module as a function of the version of program. Final value is the percentage of participates who completed endpoint data collection. Only participants who completed baseline assessment after having agreed to be randomized are included.

where elevated return rates were associated with participants returning for endpoint assessment after having finished their assigned program. No differences were found for return to the fifth session (Version 6) or completion of endpoint assessment (Version 5) $\left(\chi^{2}=0 \cdot 22, \quad \mathrm{df}=1\right.$, $p=0 \cdot 617)$.

\section{DISCUSSION}

\section{Principal findings}

The present study found that short interventions delivered over the internet can lead to an immediate small to moderate reduction of depression symptoms on post-tests in a sample of spontaneous website visitors. Not all brief interventions were found to be effective. Brief CBT (in a single module) (Version 1) is not sufficient by itself to reduce depression. Nor is a combined condition of brief CBT plus problem solving (Version 2). With the exception of the full program, versions with an extended CBT component (Module 2) were associated with greater improvement than those without this module. This finding suggests that extended CBT may be an important component in the reduction of depression symptoms. However, there are two other potential explanations. The effects may be due to spontaneous remission, with those exposed to longer duration programs having longer time to improve. A second possibility is that the content of the modules is inconsequential and that non-specific effects such as engagement are responsible for the pattern of findings. Neither of these explanations is fully consistent with the findings. If length were the important determinant, the longest program would be expected to yield the greatest improvement. This was not the case. Similarly, if engagement were the basis for the program's success, and assuming engagement were operationalized as the amount of material offered, all components (at least those of equal length) might lead to approximately equal outcomes. This was not consistent with outcomes. For example, Version 3 (Modules 1, 4 and 5) was associated with somewhat less improvement compared to Version 4 (Modules 1,2 and 5), which yielded the largest overall effect size. Moreover, the longer programs with greater opportunity for engagement were not necessarily associated with greater improvement. In short, there is some evidence to suggest that extended CBT may be an important component of effective online delivery, and that components of problem solving and stress reduction did not contribute significantly to treatment outcomes.

\section{Relationship to other relevant research studies}

The size of the effect in those who undertook at least three of the modules is somewhat lower than other education and clinical interventions for depression in clinical samples. The effect of CBT from major meta-analyses using clinical samples suggests effects in the range of 0.82 to 1.0 (Gloaguen et al. 1998; Churchill et al. 2001), while the effect sizes for successful interventions in this study were approximately $0 \cdot 40$. Effect sizes from these meta-analyses are likely to be inflated as some are estimated on outcomes for completers rather than on ITT. Given the lack of a therapist or a trial manager whose addition would have increased both retention and efficacy (Marks et al. 2003), the level of improvement achieved in the present trial is considerable. However, it is difficult to estimate with any certainty how similar web visitors are to those recruited to clinical trials. Evidence from our earlier work indicates that compared 
to the general population, internet users have high levels of depression symptoms (Christensen et al. 2002). A high proportion of participants from our earlier trials (over $50 \%$ ) met thresholds for clinical depression using cut-off values recommended for the Center for Epidemiologic Studies Depression (CES-D) scale (Randolf, 1977). The relationship between CES-D and Goldberg Depression Scale scores established in earlier trials would indicate that approximately $50 \%$ of the participants in our current trial were also above threshold and met levels of depression caseness.

Consistent with previous research showing that web-based applications have a high attrition rate even when they are brief, the majority (at least $70 \%$ ) of those who completed baseline assessment failed to complete even one module. Attrition is a result of the length of the intervention (number of modules) rather than being due to the content of specific parts of the program. It is important that attrition rates for internet interventions are considered against appropriate benchmarks. By their nature, participants in traditional RCTs have usually passed through several levels of clinical filtering. By contrast, automated trials of internet interventions feature broad and unfiltered participant catchment, high and anonymous accessibility, ease of enrolment and little personal or financial commitment, with consequent ease of drop-out (Eysenbach, 2005). Personal characteristics such as the severity of mental health problems, preference for certain conditions and expectations of website content are also likely to be important. Some individuals may specifically seek a full programmatic approach to their condition, while others are primarily looking only for information or a means of meeting specific goals or to experiment with treatment. Recognizing these multiple paths and trajectories of web usage means that low continuation and high drop-out do not necessarily denote a poor intervention.

Nevertheless, from a clinical perspective this study suggests that internet users would benefit from tracking and monitoring. Additional supportive resources are desirable to maximize mental health outcomes for individuals who want to undertake full CBT programs on the internet. Methods currently being trialed in conjunction with web applications include the use of telephone calls and email reminders for diabetes (Tate et al. 2003) and depression (Clarke et al. 2005). These appear to both increase adherence to the program and improve health outcomes. Data from previous comparisons of supported and unsupported users on MoodGYM support these findings (Christensen et al. 2004b).

\section{Research methodology}

An innovative feature of the current study is the use of a software application for recruitment, randomization and delivery of a range of interventions. Fully automatized online RCTs to evaluate research questions of this type studied here are feasible, being fast, efficient and costeffective. Paul et al. (2005) have argued for the benefits of the internet in the management of clinical trials, pointing to advantages in aspects of protocol development, patient registration, software for randomization, data collection, and data analysis. Apart from shared database management applications, these advantages include the usefulness of the internet for seamlessly integrating separate and specific tools, such as randomization software, or software applications for data analysis.

\section{Limitations of the present study}

There are a number of attributes of the study. First, the sampling frame for recruitment to the trial is effectively self-defined. Participants who volunteered to be randomized might differ substantially from those who chose not to be randomized, in unmeasured as well as measured attributes. Although the analysis used all available data in the estimation of effects, only a small proportion of individuals completed their full, assigned intervention. While the MAR assumption underlying these analyses is more permissive than the use of complete cases and has been shown to be relatively robust to violation, as the extent of attrition increases this assumption becomes more crucial in determining the results. It is also important to consider that endpoint assessments were undertaken immediately on conclusion of the assigned modules. Thus, individuals assigned to a shorter version had less time to experience spontaneous symptom remission or to consolidate benefits from early program modules. However, as noted above, the length of time since baseline cannot 
fully explain the pattern of results observed, because some longer versions of the program resulted in less change in depression scores and the longest program had effects indistinguishable from one module. Data from the Version 6 'All modules' intervention might be seen as suggestive of a toxic effect, with the positive effects of increasing exposure not observed relative to a three-module version that included two CBT modules. However, only a very small number of participants completed the full program. The length of time on the site was recorded. However, we were unable to infer the length of time spent on the program from the login times that were recorded for each module. Even as an approximation to time on task on the site, these data were highly contaminated by responses from users whose login time covered days. The design of the study is also limited by a lack of a formal control group. However, the use of an open access website precluded the inclusion of a wait-list control design for both ethical and practical reasons (there would be little chance that users would return unassisted at the end of the wait-list period). No follow-up data were available.

\section{Further research}

Further research into the components of effective CBT interventions for depression would benefit from building on the present research design. The study conditions in the present study were constrained by the structure of the website, creating difficulties in some instances to determine which aspects of the various versions of the site were crucial to outcomes and to disentangle the role of spontaneous remission, engagement and other non-specific components. The use of automated RCTs with high recruitment rates may have a unique role to play in separating both non-specific and psychotherapeutic components, and to evaluate important features of CBT packages. A theoretically driven study using a purpose-built website that held the duration of the trial constant, manipulated both content and (an operationally defined measure of) engagement is warranted.

\section{CONCLUSIONS}

Mental health problems are a leading cause of health-related economic burden in the world today and the major cause of productivity loss. According to the World Health Organization (2005), over 450 million people worldwide are affected by mental, neurological or behavioural problems at any one time, and as many as $50 \%$ of individuals do not get professional help for these problems. Unsupported, open access website interventions are capable of bringing about improvement in depression symptoms on a mass scale. This study suggests that, even allowing for attrition and moderate effect sizes, short website interventions have the potential to exert significant effects at a population level. The investigation of internet sites as 'entry points' into more formal care and the use of online tracking or monitoring of progress on internet sites warrant further research.

\section{ACKNOWLEDGEMENTS}

This study was supported by National Health and Medical Research Council (Australia) Programme Grant 179805. The work of the project is independent. We thank R. Pass, D. Berriman and C. Groves for assistance with the manuscript. Dr Dear provided assistance with the randomization algorithm.

\section{DECLARATION OF INTEREST}

H.C. and K.M. are authors of the MoodGYM site, which is offered free of charge on the internet.

\section{REFERENCES}

Christensen, H., Griffiths, K. M. \& Jorm, A. F. (2004a). Delivering interventions for depression by using the internet: randomized controlled trial. British Medical Journal 328, 265. doi:10.1136/ bmj.37945.566632.EE.

Christensen, H., Griffiths, K. M. \& Korten, A. E. (2002). Web-based cognitive behaviour therapy (CBT): analysis of site usage and changes in depression and anxiety scores. Journal of Medical Internet Research 4(1): e3.

Christensen, H., Griffiths, K. M., Korten, A. E., Brittliffe, K. \& Groves, C. $(2004 b)$. A comparison of changes in anxiety and depression symptoms of spontaneous users and trial participants of a cognitive behaviour therapy website. Journal of Medical Internet Research 6, e46. doi:10.2196/jmir.6.4.e46.

Christensen, H., Jorm, A. F., Mackinnon, A. J. M., Korten, A. E., Jacomb, P. A., Henderson, A. S. \& Rodgers, B. (1999). Age differences in depression and anxiety symptoms: a structural equation modeling analysis of data from a general population sample. Psychological Medicine 29, 325-339.

Churchill, R., Hunot, V., Corney, R., Knapp, M., McGuire, H. Tylee, A. \& Wessely, S. (2001). A systematic review of controlled trials of the effectiveness and cost effectiveness of brief 
psychological treatment for depression. Health Technology Assessment 5, 1-173.

Clarke, G., Eubanks, D., Reid, E., Kelleher, C., O'Connor, E., DeBar, L. L., Lynch, F., Nunley, S. \& Gullion, C. (2005). Overcoming depression on the Internet (ODIN) (2): a randomized trial of a self-help depression skills program with reminders. Journal of Medical Internet Research 7, e16. doi:10.2196/jmir.7.2.e16.

Eysenbach, G. (2005). The law of attrition. Journal of Medical Internet Research 7, e11. doi:10.2196/jmir.7.1.e11.

Gloaguen, V., Cottraux, J., Cucherat, M. \& Blackburn, I. M. (1998). A meta-analysis of the effects of cognitive therapy in depressed patients. Journal of Affective Disorders 49, 59-72.

Goldberg, D. P., Bridges, K., Duncan-Jones, P. \& Grayson, D. (1988). Detecting anxiety and depression in general medical settings. Psychological Medicine 17, 461-470.

Griffiths, K. M., Christensen, H., Jorm, A. F., Evans, K. \& Groves, C. (2004). Effect of a web-based depression literacy and cognitive behavioural therapy interventions on stigmatizing attitudes to depression: randomized controlled trial. British Journal of Psychiatry 185, 342-349.

Holm, J., Holm, L. \& Bech, P. (2001). Monitoring improvement using a patient-rated depression scale during treatment with anti-depressants in general practice. A validation study on the Goldberg Depression Scale. Scandinavian Journal of Primary Health Care 19, 263-266.

Marks, I. M., Mataix-Cols, D., Kenwright, M., Cameron, R., Hirsch, S. \& Gega, L. (2003). Pragmatic evaluation of computer-aided self help for anxiety and depression. British Journal of Psychiatry 183, $57-65$.

Parslow, R. A., Christensen, H., Griffiths, K. M. \& Groves, C. (2006). The warpy thoughts scale: a new 20 -item instrument to measure dysfunctional attitudes. Cognitive Behavior Therapy 35, 106-116.

Parslow, R. A., Jorm, A. F., Christensen, H. \& Rodgers, B. (2004). Use of medical services after participation in a community-based epidemiological health survey. Social Psychiatry and Psychiatric Epidemiology 39, 311-317.

Paul, J., Seib, R. \& Prescott, T. (2005). The internet and clinical trials: background, online resources, examples and issues. Journal of Medical Internet Research 7, e5. doi:10.2196/jmir.7.1.e5.

Randolf, L. S. (1977). The CES-D scale: a self-report depression scale for research in the general population. Applied Psychological Measurement 1, 385-401.

Tate, D. F., Jackvony, E. H. \& Wing, R. R. (2003). Effects of Internet behavioral counseling on weight loss in adults at risk for type 2 diabetes: a randomized trial. Journal of the American Medical Association 289, 1833-1836.

Ward, E., King, M., Lloyd, M., Power, P., Sibbald, B., Farrelly, S., Gabbay, M., Tarrier, N. \& Addington-Hall, J. (2000). Randomised controlled trial of non-directive counseling, cognitive behaviour therapy, and usual general practitioner care for patients with depression. I: clinical effectiveness. British Medical Journal 321, 1383. doi:10.1136/bmj.321.7273.1383.

World Health Organization (2005). WHO sites: Mental health (www.who.int/mental_health/en/). Accessed 10 October 2005. 